\title{
Nanomedicine - the future of cancer treatment: a review
}

\begin{abstract}
Conventional cancer therapies are limited to surgery, radiation, and chemotherapy. Conventional treatments come with significant adverse effects. Modern cancer treatments focus on precise drug delivery to the cancer tissues and minimize adverse effects on healthy cells. Researchers have been working on an improved technique for delivering chemotherapeutic agents precisely at the molecular level in the tumor tissue. It has led to the use of nanotechnology in cancer treatment. Nanotechnology is the science and engineering of controlling matter, at the molecular scale, to create devices with novel chemical, physical and biological properties. Nanoscale objects are used themselves or as part of larger devices containing multiple nanoscale objects. Almost every field of Biosciences uses nanotechnology and all of which have an impact on biomedicine. It has the potential to change the current methods to diagnose and treat cancer. There has been real progress in translating nano-based cancer therapies and diagnostics into the clinic, and much more are in development. Nanoparticles are mainly used as nanocarriers to deliver the cytotoxic drugs to the tumor tissue. Use of nanoparticles is based on different concepts of pharmacology. Nanomedicine also is utilized to deliver multiple drugs at the cancer site at the same time for a better cytotoxic effect. CytImmune Sciences is a developing field of nanomedicine for targeted chemotherapy method. They selectively deliver the drug at the cancer site because of the increased permeability of the blood vessels at the tumor site. This article reviews various nanomedicine-based cancer therapeutics.
\end{abstract}

Keywords: immunotherapy, adoptive cell transfer, nanocarriers, nanoshell, PSMA, phototherapy, PDT
Volume 8 Issue I - 2017

Roy Sebastian

Baylor Scott \& White Medical Center, USA

Correspondence: Roy Sebastian, BSW Medical CenterSunnyvale, 23I S.Collins Rd., Sunnyvale, TX 75|82 USA, Tel (2I4) 9307527,Email msoler@hospital-ribera.es

Received: May 09, 2017 | Published: May 12, 2017
Abbreviations: FDA, food and drug administration; PMSA, prostate-specific membrane antigen; siRNA, small interfering RNA; FSC, feedback system control (FSC); TNF- $\alpha$, tumor necrosis factor alpha; PEG-THIOL: thiol-derivatized polyethylene glycol

\section{Conventional cancer treatment}

Conventional cancer therapies are limited to surgery, radiation, and chemotherapy. All three methods cause damage to healthy tissues or incomplete eradication of cancer. Conventional chemotherapy suffers the lack of aqueous solubility of the drugs, lack of selectivity for the cancer cells and multidrug resistance developed after repeated administration of the same drug. ${ }^{1}$ Nanotherapeutics is a rapidly progressing field of cancer research aimed to solve several limitations of conventional drug delivery systems. The nonspecific target of cancer chemotherapy leads to the damage of rapidly proliferating normal cells. These adverse effects can be significantly reduced through the administration folate and transferrin-mediated nanotherapeutics which are aimed to target cancerous cells. ${ }^{2}$ Multidrug resistance is a challenge in cancer chemotherapy that can be managed effectively by using solid lipid nanoparticles, mesoporous silica nanoparticles, nanoparticulated chemosensitizer, nanoparticulated poloxamer, polymeric nanoparticles, and magnetic nanoparticles. ${ }^{3}$ Chemotherapeutic drugs are hydrophobic, and that leads to poor aqueous solubility and low bioavailability. It can be overcome by nanocrystals, albumin-based nanoparticles, liposomal formulation, polymeric micelles, cyclodextrin and chitosan-based nanoparticles. ${ }^{1}$ Nanotherapeutics help to overcome the lack of selectivity, multidrug resistance and lack of aqueous solubility of conventional cancer chemotherapy. Nanotechnology provides nanoparticles that target chemotherapies directly and specifically to cancerous cells and neoplasms, guides in surgical resection of tumors, and enhances the therapeutic efficacy of radiation-based and other anti-cancer treatment modalities. Use of nanotechnology-based therapeutic agent's leads to a decreased risk to the patient and an improved survival rate ${ }^{4}$ The use of nanotechnology in cancer treatment increased the possibility of destroying cancer tissues with minimal damage to healthy tissue and organs, detection of cancer and elimination of cancer cells before they form tumors. As the field of chemotherapy gradually integrates nanoparticles to deliver multiple chemotherapeutic agents with different targets, a non-conventional dosing will be necessary. Medical conditions vary from patient to patient so that the phenotypic personalized medicine will be required. Also, these issues create a parameter space that is too large to be individually tested and can result in an arbitrary dosing scenario.

\section{Modern cancer treatment}

The immune system has significant natural capacity to identify and destroy abnormal cells. This may prevent the development of many cancers but not all cancers. Some cancer cells escape the anticancer effect of the natural immune system by reducing the expression of tumor antigens on their surface, inactivating the immune cells by expression of certain proteins, or modifying the cells in the surrounding microenvironment to release substances to suppress the immune response. Immunotherapies either stimulate the activities of specific components of the immune system or counteract signals produced by cancer cells that suppress immune responses. ${ }^{5}$ The author discusses some important immunotherapy methods below. 


\section{Immune checkpoint modulators}

There are certain natural proteins called immune checkpoint proteins that normally keep immune responses in check by preventing intense responses that might damage normal cells as well as abnormal cells. Blocking the activity of immune checkpoint proteins stimulate the immune system and increase its ability to destroy cancer cells. Several immune checkpoint inhibitors have been approved by the Food and Drug Administration (FDA). The first FDA approved drug was ipilimumab for the treatment of advanced melanoma, blocks the activity of a checkpoint protein known as CTLA4, which is expressed on the surface of activated cytotoxic T lymphocytes. ${ }^{6}$ CTLA4 inactivate these $\mathrm{T}$ cells, thereby reducing the strength of immune responses; ipilimumab binds to CTLA4 and prevents it from sending its inhibitory signal.

\section{Adoptive cell transfer (ACT)}

Infiltrated T-cells from a patient's tumor are collected from samples of the tumor. The T-cells that show the greatest recognition of the patient's tumor cells in laboratory tests are selected, and large populations of these cells are grown in the laboratory. The cells are then activated by treatment with immune system signaling proteins called cytokines and infused into the patient's bloodstream.?

\section{Therapeutic antibodies and cancer treatment vaccines}

Therapeutic antibodies are made in the laboratory that are designed to destroy the cancer cells. ${ }^{5}$ One class of therapeutic antibodies, called antibody-drug conjugates (ADCs), has proven to be particularly effective to kill cancer cells. ADCs are created by chemically linking antibodies, or fragments of antibodies, to a cytotoxic substance. The antibody portion of the ADC allows it to bind to a target molecule that is expressed on the surface of cancer cells. The toxic substance can be a poison, such as a bacterial toxin; a small-molecule drug; or a radioactive compound. Once an ADC binds to a cancer cell, it is taken up by the cell and the toxic substance kills the cell. ${ }^{8}$ Cancer treatment vaccines are usually prepared from a patient's own tumor cells or from substances produced by tumor cells. ${ }^{5}$ They are designed to treat already developed cancers by strengthening the body's natural defenses against the cancer. ${ }^{9}$

\section{Chimeric antigen receptor T-cell therapy (CAR-T cell therapy)}

It is an advanced version of Adoptive Cell Transfer techniques. After collection, the $\mathrm{T}$ cells are genetically engineered to produce special receptors on their surface called chimeric antigen receptors (CARs). CARs are proteins that allow the $\mathrm{T}$ cells to recognize a specific protein (antigen) on tumor cells [10]. These engineered CAR-T cells are then grown in the laboratory until they expand their population in billions. The expanded population of CAR T-cells is then infused into the patient. After the infusion, the T cells multiply in the patient's body and, with guidance from their engineered receptor, recognize and kill cancer cells that harbor the antigen on their surfaces. ${ }^{10}$ Despite the promising clinical results, CAR-T cell therapy also involves several deleterious types of toxicity due to the inability to control $\mathrm{T}$ cell activity and some tumor-associated antigens that are presented by both diseased and healthy tissue. The prominent toxicity of CAR-T cell therapy involves cytokine release syndrome (CRS) and "on-target, off-tumor" toxicity. ${ }^{11}$

\section{Stem cell transplant}

The types of stem cell transplant includes autologous (stem cells collected from the donor before initiating high dose chemotherapy) allogeneic (stem cells from a donor is used), and syngeneic (stem cells collected from an identical sibling). The harvested marrow is filtered, stored in a special solution in bags, and then frozen. When the marrow is to be used, it's thawed and then put into the patient's blood through a vein, just like a blood transfusion. The stem cells travel to the bone marrow, where they engraft and start to make blood cells. Signs of the new blood cells usually can be measured in the patient's blood tests in about two to four weeks. ${ }^{12}$

\section{Principles of nanotechnology in cancer treatment}

\section{Nanocarriers}

Conventional chemotherapy utilizes drugs that are able to kill cancer cells effectively. But these cytotoxic drugs destroy healthy cells in addition to tumor cells, leading to many unintended adverse side effects. Nanoparticles are used as drug carriers for chemotherapeutics to deliver medication directly to the tumor while sparing healthy tissue. Nanocarriers have several advantages over conventional chemotherapy. ${ }^{13}$ Nanomaterials offer manifold advantages as drug carriers. ${ }^{4}$ Evidently, the drug particles that are large cannot reach the remote, secluded areas of the body. Because of the small size of the cell, the particles should be small enough with nanoscale dimensions to penetrate and cross the cell boundary. ${ }^{14}$ The tiny capillaries have 5-6 micron diameter, and most of the microparticles cannot pass through them. So nanoparticles are more suitable than microparticles for intravenous delivery. For systemic circulation, the particle diameters should lie in the range of $10-100 \mathrm{~nm}$ to have access to various parts of the body. ${ }^{14}$ Secondly, nanomaterials raise the drug effectiveness since they are consumed by cells efficiently than comparatively larger micromolecules. The drug is integrated into a matrix of nanoparticle or attached to the particle surface. As nanoparticles possess very high surface to volume ratio, the dissolution rate is increased. For example, when formulated as nanosuspensions, poorly soluble drugs like paclitaxel, cyclosporine, or amphotericin B exhibit an increased rate of dissolution and absorption in the gastrointestinal tract. Thirdly, nanomaterials improve the uptake of a poorly soluble drug through targeted drug delivery at the particular disease site. ${ }^{15}$ Nanoparticles are formulated to adsorb preferentially on organs or tissues depending on the particle charge, surface properties, and relative hydrophobicity, fourthly, nanomaterials help in the lessening of undesirable side effects by a controlled release. ${ }^{16}$ Nanosphere encapsulation of the drug safeguards against degradation and prolongs exposure of the drug by restricted release, a preferred quality of any chemotherapeutic agents. ${ }^{14}$ Therefore, use of nanomaterials in the pharmaceutical sector improves therapeutic index, providing solutions for delivery problems. Nanomaterial-based drug carriers can protect drugs from being degraded in the body before they reach their target, and increase bioavailability to obtain the best effect from it. ${ }^{17}$ It also enhances the absorption of drugs into tumors, and the cancerous cells themselves thus prevent drugs from interacting with normal cells and avoids side effects.

\section{Passive targeting}

Because of their size and surface properties, certain nanoparticles can escape through blood vessel walls into tissues. Nanoparticles accumulate in tumors since tumors have leaky blood vessels and defective lymphatic drainage. ${ }^{18}$ This feature enables concentrating the attached cytotoxic drug where it's needed, protecting healthy tissue and significantly reducing adverse side effects. Examples of such diseases where passive targeting of can be achieved are the tumor tissues and inflamed tissues. There are several nanocarrier-based 
drugs in the market, which rely on passive targeting through a process known as enhanced permeability and retention. ${ }^{19}$ Because of their size and surface properties, certain nanoparticles can escape through blood vessel walls into tissues.

\section{Active targeting}

Active targeting is based on the molecules expressed by cancerous cells on their surfaces and the selective targeting by nanoparticles. Attaching a molecule to a nanoparticle enables targeting of the molecule to a cell that expresses a particular receptor. Active targeting principle is used to deliver drugs into the cancerous cell, by inducing the cell to absorb the nanocarrier. ${ }^{14}$ Active targeting can be combined with passive targeting to reduce further the interaction of carried drugs with healthy tissue. Nanotechnology-enabled targeting can also increase the efficacy of a chemotherapeutic, achieving greater tumor reduction with lower doses of the drug. ${ }^{4}$

\section{Destruction from inside the cell}

Nanoshells are a form of nanoparticles that are used in the laboratory to destroy tumors from the inside by its thermal properties. Nanoshells can be designed to absorb light of different frequencies, generating heat (hyperthermia). ${ }^{20}$ The multifunctional gold nanoshells could be a more efficient cancer treatment because of its ability to target cancer cells specifically and spare healthy cells. After the nanoshells get into the cancer cells (via active targeting), scientists apply near-infrared light that is absorbed by the nanoshells. It creates an intense heat inside the cancer tissue that selectively kills the tumor cells without disturbing neighboring healthy cells. ${ }^{21}$

Newer targeted magnetic nanoparticles that are in development will be visible through Magnetic Resonance Imaging (MRI) and can destroy cells by hyperthermia. ${ }^{22}$ Features of improved targeting ability, guiding the nanoshells to specific cancer cells and attaching to markers on the surface of the cells are attributed to the small peptides situated on the surface of the nanoshells. ${ }^{23}$ Cancer cells maintain an acidic environment, and that triggers the offloading of the anticancer drugs. The specific nanostructure of the gold nanoshells could also allow near-infrared light to be absorbed and converted into heat whereby cancer cells are exposed to slightly higher temperatures than usual to destroy them. ${ }^{21}$ Some studies have demonstrated the use of iron oxide gold-shell nanoparticles that are MRI-visible photothermal sensitizers during laser irradiation of pancreatic cancer cells. ${ }^{24}$ Exposure to iron oxide gold-shell nanoparticles followed by laser irradiation led to significant improvement in the management of pancreatic cancer cell. ${ }^{24}$ There are some major disadvantages for nanoshell therapy such as drug toxicity and tumor resistance. Toxicity can cause significant complications, such as neutropenia or heart failure that necessitate cessation of treatment. The tissue damage inflicted by some nanoshell therapies can even be fatal.

In spite of all disadvantages, there are many significant benefits of the nanoshell therapy too. Nanotechnology-based therapeutics have exhibited clear advantages compared to unmodified drugs, including improved half-lives, retention, and targeting efficiency, and fewer patient side effects. ${ }^{25}$ Many nanomedicine compounds are in various stages of trials or already approved by the U.S. Food and Drug Administration (FDA). Studies showed that nanoparticles- combined chemotherapeutic drug with prostate-specific membrane antigen (PSMA) has shown to reduce lung and tonsillar lesions with greater efficacy compared with the conventional chemotherapeutic drug alone, and at substantially lower doses. ${ }^{26}$

\section{Simultaneous delivery of two drugs}

Researchers recently reported the efficacy of multidrug delivery using nanoparticles in effectively mediating resistance in relapsing cancers and to improve triple-negative breast cancer treatment. ${ }^{27}$ For example, delivery of layer-by-layer small interfering RNA (siRNA) and doxorubicin for breast cancer therapy. Others include simultaneous loading of siRNA and tumor-penetrating peptides against ovarian cancer, and sequential administration of multiple types of nanoparticles for pancreatic cancer treatment. ${ }^{28}$

For chemotherapies modified using nanotechnology to change hematological and oncological practice, the application of engineered nanomedicines must be paired with emerging strategies to design rationally nanotherapeutic combinations. This is crucial because combinatorial therapy is widely used in treating cancer and infectious diseases, and is an efficient way to simultaneously address the barriers to treatment success.

\section{Nanotechnology-based therapeutic agents in cancer treatment}

Current clinical methodologies for combinatorial drug design will be replaced as the field of chemotherapy accepts the use of nanoparticles that deliver multiple compounds with different targets, and avoids the additive dosing. Medical conditions vary from patient to patient so that the phenotypic personalized medicine will be required. Also, these issues create a parameter space that is too large to be individually tested and can result in an arbitrary dosing scenario.

Nanomedicines need to be paired with advanced strategies to determine dosing conditions that can simultaneously optimize for efficacy and safety to enable long-term permanent disease management. One promising route is the field of feedback system control (FSC), which relies on phenotypic responses instead of interrogating cellular pathways, their individual protein components, or a spectrum of genotypic responses. ${ }^{28}$ FSC utilizes outcomes to suggest new possible combinations before rapid convergence toward an optimal combinatorial dose, pharmacokinetics, and pharmacodynamics. FSC also will help personalized nanomedicine dosing on a case-by-case basis as combinations will vary from patient to patient.

\section{Nanomedicines of future}

CytImmune is one of the major companies doing research on nanomedicines in cancer treatment. CytImmune founded in 1988, has transitioned from a successful diagnostics company into a clinical stage nanomedicine company with a core focus on the discovery, development and commercialization of multifunctional, tumortargeted therapies. It is a global leader in the field of nanomedicine with the successful completion of a Phase I clinical trial of CYT-6091the first in a series of products based on the CytImmune's Aurimune nanomedicine platform (cytimmune.com). It utilizes colloidal gold nanoparticles to deliver drugs directly to cancer tumors. CytImmune uses a combination of techniques to target the nanoparticle to cancer tumors. First, the nanoparticle is designed to be too big to exit most healthy blood vessels. However, some blood vessels located at the site of tumors are leaky, allowing the nanoparticle to leave the blood vessel at the tumor site [29]. They also attach molecules of tumor necrosis factor alpha (TNF- $\alpha$ ) - a tumor-killing agent, to the nanoparticle, as well as molecules of Thiol-derivatized polyethylene glycol (PEGTHIOL). PEG-THIOL hides the TNF- $\alpha$ bearing nanoparticle from the immune system allowing the nanoparticle to flow through the blood stream without being attacked. Once the nanoparticle is in the region 
of a cancer tumor TNF molecule, bind the nanoparticle to cancer cells. CytImmune's patented technology is based on colloidal gold particles that carry specific drugs to targets such as cancer cells. ${ }^{29}$ The particles allow the drugs to be safely transported through the blood stream and directed to their specific cellular targets. There are few other targeted chemotherapy treatment and nanomedicines under development. The following Table 1 lists some important therapeutic agents. ${ }^{30}$

Table I Nanomedicines ${ }^{30}$

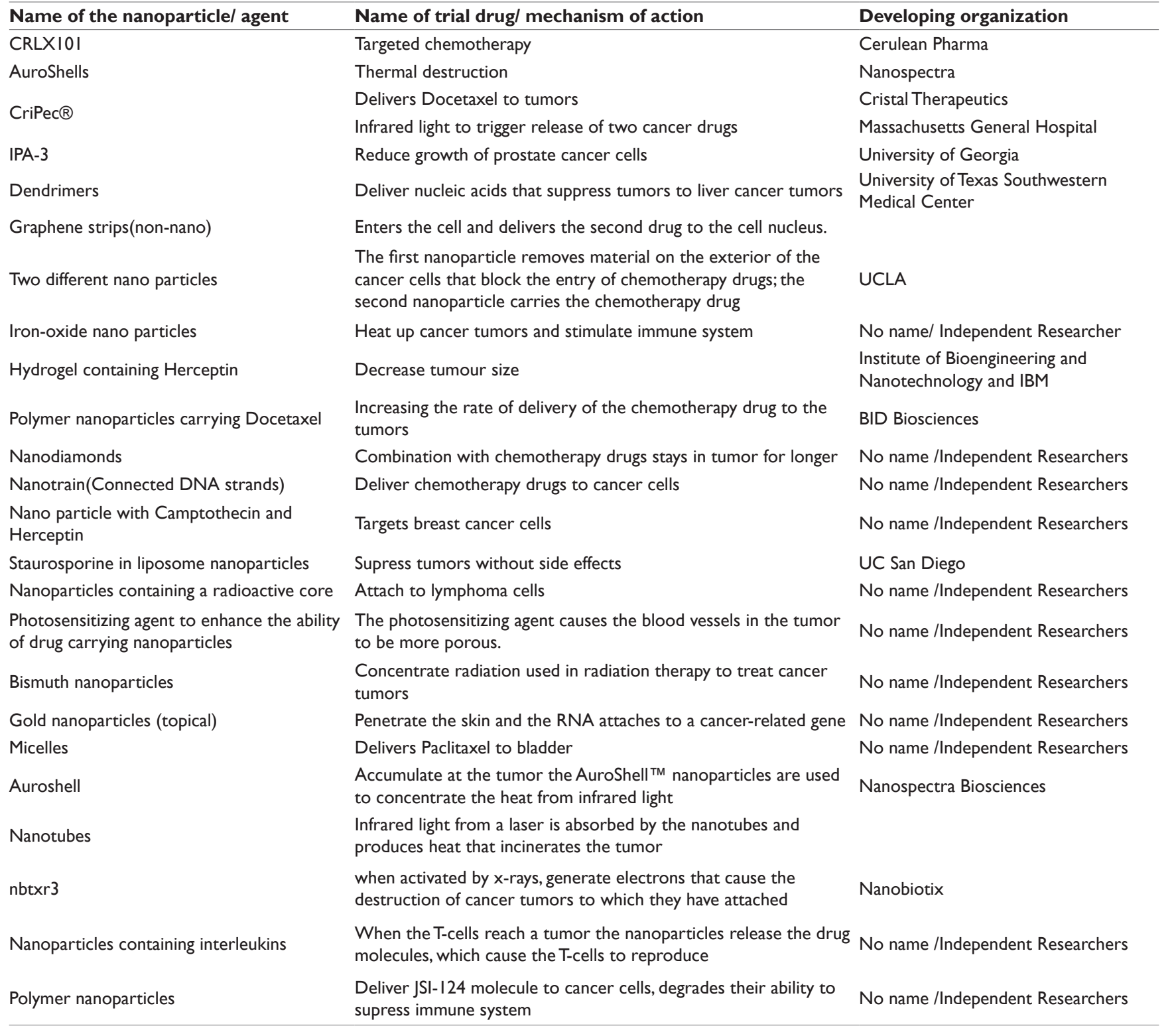

\section{Nanomedicine in early cancer detection}

Nanoparticles are not just used only to treat cancer, but are also useful for early detection of cancer. Sensors based on nanoparticles or nanowires can detect proteins related to specific types of cancer cells in blood samples. ${ }^{31}$ It allows early detection of cancer. T2 Biosystems uses superparamagnetic nanoparticles that bind to cancer indicating protein and cluster together. These clusters provide a magnetic resonance signal showing the presence of the cancer-related protein. ${ }^{32}$ One of the studies used quantum dots and molecules that emit a fluorescent glow to detect DNA strands that are early indicators of cancer. $^{33}$

\section{Conclusion}

CancerNanomedicine possesses the versatility required to overcome some of the most challenging impediments to treatment success while using conventional chemotherapeutic agents. Designing appropriate nanotherapeutic combinations using rapid convergence solutions such as Feedback System Control (FSC) represents a promising pathway from cancer management towards cancer elimination. Research should focus on identifying the appropriate nanoparticles that work well with certain chemotherapeutic agents for the drug's cytotoxic effect or the cytotoxic effect of the nanoparticles itself after activating with a suitable form of electromagnetic energy. Studies also should be encouraged to identify and formulate nanoparticle-based tests 
for early identification of cancer. Cancer studies need to hasten the clinical trials, and FDA approval to enable clinicians to use these tests in the clinics conveniently as a preventive measure.

\section{Funding}

None.

\section{Acknowledgements}

None.

\section{Conflicts of interests}

The authors declare that there is no conflict of interest.

\section{References}

1. Chidambaram M, Manavalan R, Kathiresan K. Nanotherapeutics to overcome conventional cancer chemotherapy limitations. JPPS. 2011;14(1):67.

2. Zwicke GL, Mansoori GA, Jeffery CJ. Utilizing the folate receptor for active targeting of cancer nanotherapeutics. Nano Reviews 3. 2012.

3. Ayers D, Nasti A. Utilisation of Nanoparticle Technology in Cancer Chemoresistance. Journal of Drug Delivery (2012):265691: 12.

4. Jabir NR, Tabrez S, Ashraf GM, et al. Nanotechnology-based approaches in anticancer research. International Journal of Nanomedicine. 2012;7:4391-4408.

5. Immunotherapy: Using the Immune System to Treat Cancer.

6. Fellner C. Ipilimumab (Yervoy) Prolongs Survival In Advanced Melanoma: Serious Side Effects and a Hefty Price Tag May Limit Its Use. Pharmacy and Therapeutics. 2012;37(9):503-530.

7. Perica K, Varela JC, Oelke M, et al. Adoptive T Cell Immunotherapy for Cancer. Rambam Maimonides Medical Journal. 2015;6(1): e0004.

8. Scott AM, Allison JP, Wolchok JD. Monoclonal antibodies in cancer therapy. Cancer Immunity. 2012;12:14.

9. Guo C, Manjili MH, Subjeck JR, et al.Therapeutic Cancer Vaccines: Past, Present and Future. Advances in Cancer Research. 2013;119 421475.

10. Barrett DM, Singh N, Porter DL, Grupp SA, June CH (2014) Chimeric Antigen Receptor Therapy for Cancer. Annual Review of Medicine 65: $333-347$.

11. Zhang $\mathrm{E}, \mathrm{Xu} \mathrm{H}$ (2017) A new insight in chimeric antigen receptorengineered $\mathrm{T}$ cells for cancer immunotherapy. Journal of Hematology Oncology 10(1).

12. Types of Stem Cell Transplants for Cancer Treatment.

13. Skandalis SS, Gialeli C, Theocharis AD, et al. Advances and advantages of nanomedicine in the pharmacological targeting of hyaluronan-CD44 interactions and signaling in cancer. Adv Cancer Res. 2014;123:277317.

14. Kumar Khanna V. Targeted delivery of nanomedicines. ISRN Pharmacology. 2012;571394.

15. Dreaden EC, Austin LA, Mackey MA, et al. Size matters: gold nanoparticles in targeted cancer drug delivery. Therapeutic Delivery. 2012;3(4):457-478.
16. Cai XJ, XuYY. Nanomaterials in controlled drug release. Cytotechnology. 2011;63(4):319-323.

17. Kumar A, Chen F, Mozhi A, et al. Innovative pharmaceutical development based on unique properties of nanoscale delivery formulation. Nanoscale. 2013;5(18):8307-8325.

18. Barua S, Mitragotri S. Challenges associated with Penetration of Nanoparticles across Cell and Tissue Barriers: A Review of Current Status and Future Prospects. Nano Today. 2014;9(2):223-243.

19. Greish K. Enhanced permeability and retention (EPR) effect for anticancer nanomedicine drug targeting. Methods Mol Biol. 2010;624: $25-37$.

20. Cai W, Gao T, Hong H, et al. Applications of gold nanoparticles in cancer nanotechnology. Nanotechnology, Science and Applications. 2008(1).

21. Cobley CM, Au L, Chen J, et al. Targeting Gold Nanocages to Cancer Cells for Photothermal Destruction and Drug Delivery. Expert Opinion on Drug Delivery. 2010;7(5):577-587.

22. Thomas R, Park IK, Jeong YY. Magnetic Iron Oxide Nanoparticles for Multimodal Imaging and Therapy of Cancer. International Journal of Molecular Sciences. 2013;14(8):15910-15930.

23. Yu MK, Park J, Jon S. Targeting Strategies for Multifunctional Nanoparticles in Cancer Imaging and Therapy. Theranostics. 2012;2(1): $3-44$.

24. Guo Y, Zhang Z, Kim DH, et al. Photothermal ablation of pancreatic cancer cells with hybrid iron-oxide core gold-shell nanoparticles. Int $J$ Nanomedicine. 2013;8:3437-3446.

25. Wu TT, Zhou SH. Nanoparticle-based targeted therapeutics in head-and-neck cancer. International Journal of Medical Sciences. 2015;12(2):187-200.

26. Chow EK, Ho D. Cancer Nanomedicine: From Drug Delivery to Imaging. 2013.

27. Li J, Wang Y, Zhu Y, Oupický D. Recent Advances in Delivery of DrugNucleic Acid Combinations for Cancer Treatment. J Control Releas. 2013;172(2): 589-600.

28. Jiang T, Mo R, Bellotti A, et al. Gel-Liposome-Mediated Co-Delivery of Anticancer Membrane-Associated Proteins and Small-Molecule Drugs for Enhanced Therapeutic Efficacy. Adv Funct Mater. 2014;24:22952304.

29. Jain S, Hirst DG, O'Sullivan JM. Gold nanoparticles as novel agents for cancer therapy. The British Journal of Radiology. 2012;85(1010):101113.

30. Pillai G. Nanomedicines for Cancer Therapy: An Update of FDA Approved and Those under Various Stages of Development. SOJ Pharm Pharm Sci. 2014;1(2):13.

31. Choi YE, Kwak JW, Park JW. Nanotechnology for Early Cancer Detection. Sensors (Basel, Switzerland). 2010;10(1):428-455.

32. Zhang Y, Wang TH. Quantum Dot Enabled Molecular Sensing and Diagnostics. Theranostics. 2012;2(7): 631-654.

33. Fang M, Peng C, Pang DW, et al. Quantum Dots for Cancer Research: Current Status, Remaining Issues, and Future Perspectives. Cancer Biology Medicine. 2012;9(3):151-163. 